\title{
Cirugía cardíaca en pacientes infectados por virus de inmunodeficiencia humana
}

\author{
Cecilia Muñoz ${ }^{A}$, Óscar Corsia ${ }^{a}$ Carlos Pérez ${ }^{B}$, Ricardo Zalaquett. $^{A}$ \\ A. División de Enfermedades Cardiovasculares. Facultad de Medicina. Pontificia Universidad Católica de Chile \\ B. Departamento de Enfermedades Infecciosas del Adulto. Facultad de Medicina. Pontificia Universidad Católica de Chile \\ a. Interno de Medicina, Facultad de Medicina. Pontificia Universidad Católica de Chile. Santiago - Chile. \\ División de Enfermedades Cardiovasculares. Facultad de Medicina. Pontificia Universidad Católica de Chile. Santiago - Chile. \\ ${ }^{*}$ Financiamiento: propio \\ * Conflictos de interés: ninguno
}

Resumen: La infección del virus de inmunodeficiencia humana (VIH) se adquiere principalmente a través del contagio sexual y los pacientes infectados siguen aumentando en el mundo. El tratamiento antirretroviral (TARV) moderno es capaz de suprimir la replicación viral y mejorar el recuento de linfocitos T CD4+, aumentando la supervivencia de los pacientes. De esta manera, aumenta el riesgo de presentar enfermedades crónicas siendo las cardiovasculares las más frecuentes.

Objetivo: Comunicar nuestra experiencia en pacientes con infección por VIH sometidos a cirugía cardiovascular.

Pacientes y Método: Estudio retrospectivo en que se revisó la base de datos del Servicio de Cirugía Cardiovascular entre los años 2009 y 2015. Identificamos a los pacientes con infección por VIH sometidos a cirugía cardiovascular.
Resultados: Catorce pacientes presentaban esa condición. La mayoría estaba en control y con TARV (11 casos) logrando que la carga viral fuera indetectable. La principal vía de infección fue la sexual (13/14 casos). Durante la cirugía se tomaron las precauciones universales de protección para el equipo quirúrgico, las que fueron efectivas en todos los casos. La enfermedad más frecuentemente tratada fue la cardiopatía coronaria (9 casos), seguido de valvulopatías severas (4 casos) en que solo uno presentaba Endocarditis Infecciosa. No hubo mortalidad asociada al procedimiento ni en el seguimiento a tres años de la cirugía. Conclusión: Los pacientes con infección por VIH están expuestos a desarrollar enfermedades cardiovasculares que requieren de tratamiento quirúrgico. Estos pueden ser realizados en forma segura con gran beneficio en su calidad de vida y de su supervivencia.

\section{Correspondencia:}

Dra. Cecilia Muñoz C. Profesor Asociado Adjunto.

División de Enfermedades Cardiovasculares

Pontificia Universidad Católica de Chile

Dirección: Marcoleta 367.

Teléfonos: 56223543231 - 084499636

mcmunoz@med.puc.cl 


\section{Cardiac Surgery in HIV positive patients}

Infection with human immunodeficiency virus (HIV+) is primarily acquired through sexual transmission and the number of infected people continues to increase. Anti-retroviral therapy (HAART) suppress viral replication and improves CD4 T cell count, increasing survival and the risk of developing chronic diseases. Cardiovascular disease is prevalent among these patients.

Objective: To report our experience in patients positive for HIV undergoing cardiovascular surgery.

Patients and Methods: We reviewed the Cardiovascular Surgery Service database from 2009 to 2015 to identify HIV-infected patients that underwent cardiovascular surgery.

Results: There were 14 HIV positive patients. Most of them were receiving HAART and the viral load was undetectable in 11 patients Thee main route of infection was sexual (13/14 cases). Universal precautions to protect the surgical team were taken during surgery. These were effective in all cases. Coronary artery disease was the most common condition treated ( 9 cases), followed by severe valve disease (4). Only one patients had infective endocarditis. There was no mortality associated with the procedure. Al patients were alive and asymptomatic 3 years after surgery.

Conclusion: Patients with HIV infection are likely to develop cardiovascular diseases requiring surgical treatment. These can be performed safely with great benefit in quality of life and survival.

Key words: Cardiac surgery; acquired immunodeficiency syndrome. 


\section{Introducción:}

En la década de los ' 80 se conoció la infección por el virus de la inmunodeficiencia humana (VIH/SIDA), la que se adquiere a través de los fluidos corporales. La vía más importante de contagio es la sexual. Inicialmente, la infección causó la muerte de todos los contagiados, por lo que durante la década de los 80 y 90 se discutió mucho sobre los riesgos y beneficios de intervenir quirúrgicamente a los pacientes con enfermedad avanzada (estados $\mathrm{B}$ y C). El temor de adquirir la letal enfermedad, a través del contacto con los pacientes o de la manipulación de sangre o fluidos llevó a que muchos cirujanos se rehusaran a tratar a estos pacientes ${ }^{1}$.

Sin embargo, se operaron pacientes por la gravedad de sus lesiones, siendo la endocarditis infecciosa complicada la principal causa. Los resultados de esas cirugías fueron muy insatisfactorios por no haber tratamientos efectivos que controlaran la sepsis en condiciones de inmunosupresión ${ }^{2}$.

En los siguientes 20 años, los procedimientos cardícos en estos pacientes, fueron excepcionales, centrándose la discusión en si los pacientes podían tolerar una cirugía abierta con circulación extracorpórea por la alta incidencia de complicaciones postoperatorias ${ }^{3,4}$. No obstante, los avances en el tratamiento médico de los pacientes con VIH permitieron mejorar los resultados de la cirugía cardíaca aumentando la referencia de pacientes para estos procedimientos 5

Actualmente, la infección por VIH no es letal. La terapia antirretroviral (TARV) efectiva permite que los pacientes vivan con el virus de la inmunodeficiencia (PVVI) con una excelente supervivencia, igual a la de la población general ${ }^{6}$. Sin embargo, estos pacientes tienen una mayor probabilidad de desarrollar enfermedades cardiovasculares, ya sea por sus hábitos, factores de riesgo y por los efectos colaterales de las drogas utilizadas para el control de la enfermedad ${ }^{7}$.

Los procedimientos cardiovasculares son relativamente frecuentes en la población general y sus resultados son muy satisfactorios en el largo plazo. Estos beneficios también se logran en PVVI, ya que los resultados inmediatos de la cirugía están determinados por la condición clínica al momento de la intervención y el procedimiento quirúrgico y no por la infección por $\mathrm{VIH}^{8}$.

En Chile, los primeros casos de infección por VIH fueron notificados en 1984. En el transcurso de los años aumentaron rápidamente alcanzando en 2013 a más de 31.000 pacientes infectados. Considerado como un problema de salud prioritario, la infección por VIH se incluyó dentro de las primeras patologías con Garantías Explícitas de Salud en el año 2010 (GES) ${ }^{9}$.

En la transmisión de la enfermedad la vía sexual es la más importante, siendo los homosexuales los más afectados (99\% de los casos). La transmisión por lesiones corto punzantes es baja (menor a $0,5 \%$ por exposición). Si el tratamiento antirretroviral es bien llevado por el paciente con VIH+ y su carga viral en plasma es indetectable, la probabilidad de transmitir la enfermedad por esta vía es aún más baja ${ }^{10,11}$. Para reducir el riesgo de infección, al manipular material contaminado o fluidos corporales en procedimientos, se recomienda el uso de doble guante ${ }^{12}$. El objetivo del presente estudio fue analizar las indicaciones y resultados de los procedimientos quirúrgicos cardiovasculares en pacientes portadores del virus de inmunodeficiencia adquirida.

\section{Pacientes y Método}

Este estudio fue aprobado por el Comité Ético Científico - de la Facultad de Medicina de la Pontificia Universidad Católica de Chile (CEC MED UC, proyecto número 160518002).

Revisamos la base de datos de la División de Enfermedades Cardiovasculares de la Red de Salud UC-CHRISTUS para identificar a los pacientes con infección por VIH sometidos a cirugía cardíaca. Se revisaron fichas clínicas, protocolos operatorios y exámenes de laboratorio.

Los pacientes que fueron operados con circulación extracorpórea (CEC) esta se realizó bajo anestesia general, monitoreo hemodinámico invasivo, anticoagulación con heparina para un tiempo de coagulación activado (ACT) mayor a 480 segundos, en hipotermia moderada y protección miocárdica con cardioplegia cristaloide.

Dentro del estudio pre-operatorio para cirugía cardíaca, la pesquisa de infección por VIH no está incluida. En nuestro país, la ley sólo permite la detección de VIH ante algunas situaciones específicas para resguardar la confidencialidad de las personas (donación de sangre u órganos, necesidad de diálisis o por solicitud del interesado $)^{13}$. Por esto, en beneficio del paciente y del personal de salud, las medidas de prevención de riesgo de contaminación durante las cirugías son las universalmente conocidas. En los casos en que se produce un accidente corto punzante se realiza la pesquisa de la infección por VIH en el paciente y en el accidentado ${ }^{14}$.

En los pacientes con VIH+ conocido, las medidas de protección para el equipo de salud fueron la información adecuada, eliminación efectiva de objetos corto punzantes, equipo de protección personal y dispositivos de se- 
guridad. El uso de doble guante quedó a discreción del operador siendo utilizado por la mayoría de los miembros del pabellón ${ }^{11}$.

La supervivencia de los pacientes se certificó a través del "Servicio de Registro Civil e Identificación de Chile".

\section{Resultados}

Pacientes: Entre los años 2009 y 2015, catorce pacientes infectados con VIH fueron sometidos a algún procedimiento quirúrgico cardíaco, lo que correspondió al $0,54 \%$ de los pacientes adultos operados en ese período. En la Figura 1 se muestra la distribución de los pacientes según el año de la intervención.

Figura 1. Número de pacientes con $\mathrm{VIH}+$ intervenidos según año de operación.

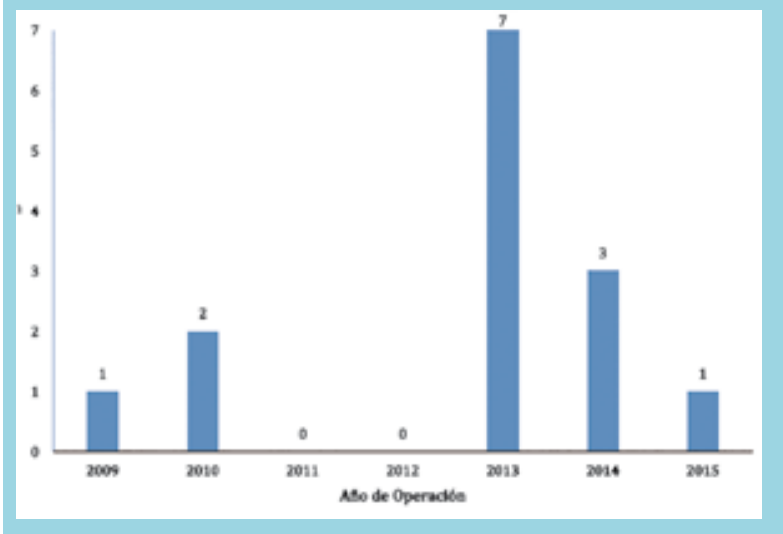

Todos los pacientes fueron hombres con edad promedio de $54 \pm 8$ años (rango 42-66 años).

En la Tabla 1 se indican las características de los pacientes. Los pacientes con enfermedad coronaria fueron de mayor edad que los con enfermedad valvular (promedio 58 vs 49 años) y llevaban más años de tratamiento médico por VIH+ (9 vs 7 años).

El factor de riesgo cardiovascular más importante fue el tabaquismo (12/14), seguido por la dislipidemia (4/14).

La vía de infección fue sexual en trece pacientes (93\%). El paciente restante negó el contagio sexual y lo atribuyó a punción con material contaminado.

La mayoría de los pacientes conocían su condición de VIH+ (12/14, 86\%) y sólo uno no se trataba.

En los pacientes con VIH en tratamiento (11) el tiempo promedio desde el diagnóstico de $\mathrm{VIH}+$ hasta la intervención cardiovascular fue $8 \pm 5,6$ años (rango 6 meses a 20 años). El recuento promedio de linfocitos T CD4+ fue

\begin{tabular}{|c|c|c|c|c|}
\hline $\begin{array}{l}\text { Indicación de } \\
\text { la intervención }\end{array}$ & $n$ & Edad & $\begin{array}{l}\text { Intervalo entre } \\
\text { diagnóstico de } \\
\text { la enfermedad } \\
\text { y cirugía }\end{array}$ & $\begin{array}{l}\text { Morbilidad } \\
\text { asociada }\end{array}$ \\
\hline $\begin{array}{l}\text { Enfermedad } \\
\text { coronaria severa }\end{array}$ & 8 & $\begin{array}{l}58 \pm 7,2 \text { años } \\
\text { (mediana 60, } \\
\text { rango 47-66) }\end{array}$ & $\begin{array}{c}9,3 \pm 6 \text { años } \\
\text { (6 meses - } \\
20 \text { años) }\end{array}$ & $\begin{array}{l}\text { Tabaquismo (7) } \\
\text { Dislipidemia (3) } \\
\text { Hipertensión Arterial (2) }\end{array}$ \\
\hline $\begin{array}{l}\text { Enfermedad } \\
\text { valvular severa }\end{array}$ & 3 & $\begin{array}{l}49 \pm 6,4 \text { años } \\
\text { (mediana 52, } \\
\text { rango 42-54) }\end{array}$ & 7 años & $\begin{array}{l}\text { Tabaquismo (2) } \\
\text { Virus Hepatitis B (1) } \\
\text { Dislipidemia (1) }\end{array}$ \\
\hline $\begin{array}{l}\text { Endocarditis } \\
\text { Infecciosa y falla } \\
\text { cardíaca aguda }\end{array}$ & 1 & 42 & 3 años & Tabaquismo \\
\hline $\begin{array}{l}\text { Pseudoaneuris- } \\
\text { ma micótico del } \\
\text { arco complicado }\end{array}$ & 1 & 55 & 0 & $\begin{array}{l}\text { Diabetes Mellitus } \\
\text { Tabaquismo } \\
\text { Alcoholismo } \\
\text { Drogas ilícitas } \\
\text { (cocaína, marihuana) }\end{array}$ \\
\hline $\begin{array}{l}\text { Neumonía } \\
\text { bilateral grave }\end{array}$ & 1 & 46 & 0 & Tabaquismo \\
\hline Total & 13 & $54 \pm 8$ años & $8 \pm 5,6^{*}$ & \\
\hline
\end{tabular}

*Incluye sólo pacientes con diagnóstico pre-operatorio.

$576 \pm 269$ céls/mm3 (rango 198 a 1163 céls. /mm3). En todos la carga viral fue no detectable.

Ocho pacientes debutaron con cuadros clínicos de enfermedad coronaria después de 9 años de TARV en promedio. Sus factores de riesgo cardiovascular fueron tabaquismo (6/8), dislipidemia (3/8) e hipertensión arterial (2/8). La intervención fue realizada en el curso de una complicación de la enfermedad coronaria en todos los pacientes, 5 con infarto agudo al miocardio, 2 con angina inestable y uno con shock cardiogénico.

Los tres pacientes restantes consultaron por síntomas de insuficiencia cardíaca. Ellos llevaban en promedio 7 años de TARV y el estudio cardiovascular demostró que presentaban enfermedad valvular grave ( 2 mitral y 1 aórtica) con indicación de manejo quirúrgico. El estudio complementario descartó enfermedad coronaria.

De los 3 pacientes con VIH sin tratamiento, sólo uno sabía que estaba infectado. En los otros dos, la enfermedad se pesquisó después de la intervención cardiovascular en el estudio requerido para instaurar Terapia de Reemplazo Renal por insuficiencia renal aguda.

\section{Indicación del procedimiento y cirugía realizada:}

Los pacientes con enfermedad coronaria fueron los más frecuentemente intervenidos (9/14 casos). La mayoría 
se operaron durante un episodio coronario agudo (5 cursando un infarto, 2 con angina inestable y 2 en shock cardiogénico). Se realizó revascularización miocárdica completa en todos los casos, en 2 asociados a otros procedimientos (anuloplastía mitral y reparación de pseudo-aneurisma micótico del arco).

La patología valvular fue la segunda causa de cirugía cardiovascular (4/14). Tres pacientes consultaron por disnea cuyo estudio demostró una valvulopatía severa. Uno con insuficiencia mitral severa por enfermedad de Barlow que se trató con reparación valvular. Otro, presentaba una estenosis aórtica severa en válvula bicúspide que fue tratado con reemplazo valvular. El tercer paciente tenía una disfunción de prótesis mitral mecánica que requirió el reemplazo de la prótesis. El cuarto paciente debutó con falla cardíaca y sepsis secundaria a endocarditis infecciosa de la válvula tricúspide y en él se efectuó un reemplazo valvular con prótesis biológica.

El último paciente intervenido, presentaba una insuficiencia respiratoria grave refractaria que requirió oxigenación con membrana extracorpórea (ECMO).

En todos los procedimientos las medidas de protección fueron efectivas no reportándose ningún accidente corto punzante.

Mortalidad: No hubo mortalidad.

Morbilidad: Los dos 2 pacientes en que el diagnóstico de $\mathrm{VIH}+$ fue post procedimiento sufrieron complicaciones. En uno se realizó cirugía endovascular del arco aórtico por un pseudo-aneurisma micótico asociada a revascularización miocárdica, se complicó con infarto cerebral, neumonía asociada a ventilación mecánica, insuficiencia renal aguda que requirió TRR. El segundo paciente fue quien presentaba insuficiencia respiratoria grave y apoyo de ECMO, que presentó insuficiencia renal, traqueostomía por ventilación mecánica prolongada e hipotonía de paciente crítico.

Seguimiento: se confirmó la supervivencia del $100 \%$ de los pacientes $3,4 \pm 1,7$ años después de la operación (rango 1 a 7 años).

\section{Discusión}

La infección por VIH es cada vez más frecuente. En Estados Unidos más de 1,2 millones de personas la padecen, pero sólo $16 \%$ tiene conocimiento de ella ${ }^{15}$. En Chile, la tasa de notificación por SIDA aumentó de 0,1 hasta 6 casos por 100.000 habitantes en el año 2011. También aumentó la notificación en etapa VIH llegando a 9,6 por cien mil habitantes en el año $2011^{16}$.

Con las terapias actuales la supervivencia llega casi el
$90 \%$ a los 5 años. Estos pacientes tienen tasas más altas de enfermedad cardiovascular que la población general, pudiendo requerir en algún momento de su evolución una intervención cardiovascular ${ }^{17}$. Muchas publicaciones de resultados de cirugía cardíaca en este tipo de pacientes, se refieren a casos clínicos o series pequeñas. Castillo, de Mount Sinai Hospital en Nueva York, reportó una de las más grandes experiencias actuales ${ }^{12}$. En más de 7.000 pacientes operados, 223 presentaban inmunosupresión $(3,1 \%)$ y solo 39 por VIH+ $(0,5 \%)$. En esa serie la mayoría fueron hombres (77\%). Kumar, en India, reporta la experiencia quirúrgica en 7 pacientes con $\mathrm{VIH}+$, todos hombres. Cinco pacientes presentaban enfermedad cardíaca y 2 fueron operados por pericarditis tuberculosa $(2008)^{18}$. Cipriano, de Texas, reporta 5 casos de pacientes operados, 4 por endocarditis infecciosa $(2000)^{19}$. Namai, de Japón, reporta 3 pacientes hombres operados, 2 por patología valvular adquirida y uno por endocarditis infecciosa $(2008)^{20}$.

En Estados Unidos, el porcentaje de pacientes con VIH+ que fueron intervenidos de cirugía cardíaca aumentó significativamente desde $0,09 \%$ en 1998 a $0,23 \%$ en el año $2009^{21}$. Las principales indicaciones de cirugía fueron enfermedad coronaria (38\%), derrame pericárdico (23\%) y patología valvular aislada (16\%). La mortalidad de los operados fue de $7,2 \%$, siendo mayor para los procedimientos pericárdicos $(17,6 \%)$ que para las cirugías cardíacas con CEC $(3,6 \%)$.

Nuestra serie está compuesta solo por hombres que en su gran mayoría adquirieron la enfermedad a través de contacto sexual. Solo 2 no tenían diagnóstico previo de infección por VIH y la gran mayoría estaba bajo TARV y carga vital no detectable. La enfermedad coronaria severa fue la enfermedad más frecuente que requirió de tratamiento quirúrgico y que se diagnosticó después de 9 años de TARV por una complicación de la enfermedad isquémica.

A pesar de que las intervenciones cardiovasculares fueron complejas, con necesidad de implantes de prótesis o dispositivos vasculares, no hubo mortalidad en esta serie y las complicaciones mayores solo se registraron en 2 pacientes. Ante la eventualidad de sufrir complicaciones infecciosas de los dispositivos, es muy importante mantener en seguimiento a los pacientes, tanto desde el punto de vista cardiaco como infeccioso. Fundamental es la adherencia a TARV y el control factores de riesgo para lograr una adecuada calidad de vida y buena supervivencia.

En procedimientos quirúrgicos, la probabilidad de ad- 
quirir infecciones a través de la manipulación de material biológico contaminado por el operador es baja. Existe el riesgo de contagio de manera cruzada de virus como el de la inmunodeficiencia y de la hepatitis B o $\mathrm{C}$, por lo que las medidas de protección deben ser utilizadas en todo momento ${ }^{9}$. También, se debe insistir en la necesidad de declarar los accidentes corto punzante para hacer el seguimiento y la prevención de graves enfermedades ${ }^{22}$.

\section{Conclusión:}

En pacientes con VIH+, la probabilidad de adquirir enfermedades cardiovasculares con repercusión severa en la función cardíaca que requieran de una intervención quirúrgica es mayor que en la población general. En esta población, los procedimientos quirúrgicos son realizados en forma segura con muy buenos resultados inmediatos y a largo plazo $21,22,23$.

\section{Referencias}

1. ABEL RM. RISK IS TOO HIGH IN AIDS PATIENT SURGERY. The New York Times, Opinion. November 9, 1987. Disponible en www.nytimes.com/1987/11/09/opinion/1-riskis-too-high-in-aids-patient-surgery-896987.html

2. FRATER RW, SISTO D, CONDIT D. Cardiac surgery in human immunodeficiency virus (HIV) carriers. Eur J Cardiothorac Surg. 1989; 3: 146-50; discussion 150-1.

3. PENNINGA, L., LAHPOR, J. R., \& VAN DER WIEKEN, L. R. (2002). Successful aortic valve replacement in a patient with AIDS. Neth Heart J. 2002; 10: 283-285.

4. TRACHIOTIS GD, ALEXANDER EP, BENATOR D, GHARAGOZLOO F. Cardiac surgery in patients infected with the human immunodeficiency virus. Ann Thorac Surg. 2003; 76: $1114-8$.

5. FILSOUFI F, SALZBERG SP, HARBOU KT, NEIBART E, ADAMS DH. Excellent Outcomes of Cardiac Surgery in Patients Infected with HIV in the Current Era. Clin Infect Dis. 2006 Aug 15; 43: 532-6.
6. D'ARMINIO MONFORTE A, SABIN CA, PHILLIPS A, STERNE J, MAY M, JUSTICE A, DABIS F, GRABAR S, LEDERGERBER B, GILL J, REISS P, EGGER M; Antiretroviral Therapy Cohort Collaboration. The Changing Incidence of AIDS Events in Patients Receiving Highly Active Antiretroviral Therapy. Arch Intern Med. 2005; 165: 416-423.

7. GOPAL, M., BHASKARAN, A., KHALIFE, W. I., \& BARBAGELATA, A. (2009). Heart Disease in Patients with HIV/ AIDS-An Emerging Clinical Problem. Curr Cardiol Rev. 2009; 5: 149-154.

8. CASTILLO JG, ADAMS DH, RAHMANIAN PB, FILSOUFI F. Cirugía cardiovascular en pacientes con VIH: epidemiología, indicaciones actuales y resultados a largo plazo. Rev Esp Cardiol. 2008; 61: 480-6.

9. MINISTERIO DE SALUD, GUÍA CLÍNICA AUGE "Síndrome de la Inmunodeficiencia Adquirida VIH/SIDA". MINSAL. $20101^{\circ}$ Edición - $20132^{\circ}$ Edición. Disponible en www. sidachile.cl/guias/GPCVIH

10. PURO V, DE CARLI G, SCOGNAMIGLIO P, PORCASI R, 
IPPOLITO G; Studio Italiano Rischio Occupazionale HIV. Risk of HIV and other blood-borne infections in the cardiac setting: patient-to-provider and provider-to-patient transmission. Ann N Y Acad Sci. 2001; 946: 291-309.

11. HENDERSON DK, FAHEY BJ, WILLY M, SCHMITT JM, CAREY K, KOZIOL DE, LANE HC, FEDIO J, SAAH AJ. Risk for Occupational Transmission of Human Immunodeficiency Virus Type 1 (HIV-1) Associated with Clinical Exposures: A Prospective Evaluation. Ann Intern Med. 1990; 113: 740-746.

12. AROWOLO O, AGBAKWURU E, OBONNA G, ONYIA C, AKINKUOLIE A, OLAOGUN J. SAFETY OF THE SURGEON: 'Double-gloving' during surgical procedures. Southern African Journal of HIV Medicine, 2014; 15: 144-147.

13. GUÍA CLÍNICA AUGE VIH/SIDA 2013 - Sida Chile. Serie Guías Clínicas MINSAL 2009disponible en www.sidachile. cl/guias/GPCVIH

14. Bravo T. GUÍA PREVENTIVA DE RECOMENDACIONES PARA TRABAJADORES (AS) SANITARIOS EN MANEJO DE MATERIAL CORTOPUNZANTE. Disponible en www. ispch.gov.cl/sites/default/files/u5/Guia_Preventiva_Cortopunzantes

15. NATIONAL CENTER FOR HIV/AIDS, Viral Hepatitis, STD, and TB Prevention. Division of HIV/AIDS Prevention. HIV in the United States: At A Glance. Disponible enhttp://www. cdc.gov/hiv/statistics/overview/ataglance.html actualizado en Julio 2015.

16. MINISTERIO DE SALUD DE CHILE. Informe Nacional: evolución de la infección por VIH/SIDA Chile 1984-2012. Departamento de Epidemiología. División de Planificación
Sanitaria. Rev. chil. infectol. 2015; 32, suppl.1.

17. CERRATO E, CALCAGNO A, D'ASCENZO F, BIONDI-ZOCCAI G, MANCONE M, GROSSO MARRA W, et al. Cardiovascular disease in HIV patients: from bench to bedside and backwards.Open Heart. 2015; 2: e000174.

18. KUMAR N, REDDY B, JITENDRA V, KUMAR M. Cardiac surgery in HIV positive patients: Growing needs and concerns. Single centre experience in an Indian setting. Ind J Thorac Cardiovasc Surg 2008; 24: 5-9.

19. ABAD C, CÁRDENAS MA, JIMÉNEZ P, ARMAS M, BETANCOR, P. Cardiac surgery in patients infected with human immunodeficiency virus. Texas Heart Institute Journal, 2000; 27: 356-360.

20. NAMAI A, SAKURAI M, AKIYAMA M. Cardiac surgery in three patients infected with the human immunodeficiency virus. Gen Thorac Cardiovasc Surg. 2008; 56: 465-467.

21. ROBICH M, SCHILTZ N, JOHNSTON D, MICK S, TSE W, $\mathrm{KOCH} \mathrm{C}$, et al. Outcomes of patients with human immunodeficiency virus infection undergoing cardiovascular surgery in the United States. JThorac Cardiovasc Surg. 2014; 148: 3066-73.

22. RASOOL F, LONE R, RASOOL I, SHAH S, SHAH M, RASOOL I, et al. Surgeon and human immunodeficiency virus infection. Int J Health Sci (Qassim). 2009; 3: 253-255.

23. RODGER A, LODWICK R, SCHECHTER M, DEEKS S, AMIN J, GILSON R, et al. Mortality in well controlled HIV in the continuous antiretroviral therapy arms of the SMART and ESPRIT trials compared with the general population. AIDS 2013; 27: 973-979. 